\title{
Robotics and Intelligent Systems Against a Pandemic
}

\author{
Alaa Khamis ${ }^{1}$, Jun Meng ${ }^{2}$, Jin Wang ${ }^{3}$, Ahmad Taher Azar ${ }^{4}$, \\ Edson Prestes ${ }^{5}$, Howard $\mathrm{Li}^{6}$, Ibrahim A. Hameed ${ }^{7}$, Árpád \\ Takács $^{8}$, Imre J. Rudas ${ }^{8}$, and Tamás Haidegger ${ }^{8,9}$ \\ ${ }^{1}$ General Motors Canada, 500 Wentworth St W, Oshawa, ON L1J 6J2, \\ alaa.khamis@gm.com \\ ${ }^{2}$ College of Electrical Engineering, Zhejiang University, YuQuan Campus, \\ No.38 ZheDa Road, HangzhouZhejiang, 310027, China, junmeng@ zju.edu.cn \\ ${ }^{3}$ Robotics Institute, Zhejiang University, YuQuan Campus, No.38 ZheDa \\ Road,Hangzhou 310027,China, dwjcom@zju.edu.cn \\ ${ }^{4}$ Faculty of Computers and Artificial Intelligence, Benha University, Benha, \\ Egypt and College of Computer and Information Sciences, Prince Sultan \\ University Riyadh, Saudi Arabia, ahmad.azar@fci.bu.edu.eg, aazar@psu.edu.sa \\ ${ }^{5}$ Informatics Institute, Federal University of Rio Grande do Sul, \\ edson.prestes@ieee.org \\ ${ }^{6}$ Dept. of Electrical and Computer Engineering, University of New Brunswick, \\ Canada, howard@unb.ca \\ ${ }^{7}$ Dept. of ICT and Natural Sciences, Norwegian University of Science and \\ Technology, Larsgårdsvegen 2, $6009{ }^{\circ}$ Alesund, Norway,ibib@ntnu.no \\ ${ }^{8}$ Antal Bejczy Center for Intelligent Robotics, EKIK, Óbuda University, \\ Budapest, HU, arpad.takacs@irob.uni-obuda.hu \\ ${ }^{9}$ Austrian Center for Medical Innovation and Technology (ACMIT), \\ Viktor-Kalpan-str.2., Wiener Neustadt, A-2700, AT, tamas.haidegger@acmit.at
}

Abstract: The outbreak of the novel coronavirus and its disease COVID-19 presents an unprecedented challenge for humanity. Intelligent systems and robotics particularly are helping the fight against COVID-19 several ways. Potential technology-driven solutions in this accelerating pandemic include, but are not limited to, early detection and diagnosis, assistive robots, indoor and outdoor disinfection robots, public awareness and patrolling, contactless last-mile delivery services, micro- and nano-robotics and laboratory automation. This article sheds light on the roles robotics and automation can play in fighting this disastrous pandemic and highlights a number of potential applications to transform this challenge into opportunities. The article also highlights the ethical implications of robotics and intelligent systems during the emergency side and in the post-pandemic world.

Keywords: Service robotics at COVID-19; coronavirus response; assistive robots; lastmile delivery; laboratory automation 


\section{Introduction}

Since the outbreak of the novel coronavirus (SARS-CoV-2), the global Robotics and Automation (R\&A) community has quickly mobilized and gathered to offer solutions to help preventing, treating and monitoring the pandemic and its effects. Contributions have materialized in several novel applications and services. Identifiying key challenges faced by health care responders and the general population remains a priority, unraveling the role of robotics in infectious disease crises [1]. According to the World Health Organization (WHO) the top priorities in the fight against COVID-19:

1. Early diagnosis;

2. Improved hygiene \& disinfection;

3. Social distancing.

The role that robotics and automation ( $R \& A)$ can play accordingly is getting well recognized, e.g., the WHO-China Joint Mission COVID-19 report cited Artificial Intelligence (AI) and robotics as key elements of the efficient response [2]. The European Commission launched an initiative (managed by the European AI Alliance) to collect ideas about deployable AI and robotics solutions, as well as information on other initiatives that could help solve the ongoing coronavirus crisis: AI-Robotics vs COVID-19 (tinyurl.com/COVID19-AI). The IEEE and other professional organizations are also taking part in the global response; e.g., working on applying the lessons learned to COVID-19 in conjunction with the IEEE Robotics and Automation Society (RAS) Special Interest Group on Humanitarian Technology (SIGHT).

This article describes a number of current and potential future applications of R\&A in the battle against the novel coronavirus and its COVID-19 disease. AI solutions are covered in other publications [3, 4]. Applications include-but are not limited to-specific solutions aligned with the WHO priorities (Fig. 1):

1. Telehealth and physical human-robot interaction systems enabling healthcare workers to remotely diagnose and treat patients;

2. Autonomous or teleoperated robots for hospital disinfection and disinfection of public spaces;

3. Social robots for families interacting with patients or with relatives in nursing homes and robots used by public safety and public health departments for quarantine enforcement and public service announcements;

4. General workflow improvements, such as hospital and laboratory supply chain robots for handling and transportation of samples and contaminated materials and robots enabling or assisting humans to return to work or companies to continue to function.

Ethical implications of R\&A during the pandemic and in the post-pandemic world are also discussed in the light of the IEEE Global Initiative. Beyond the 


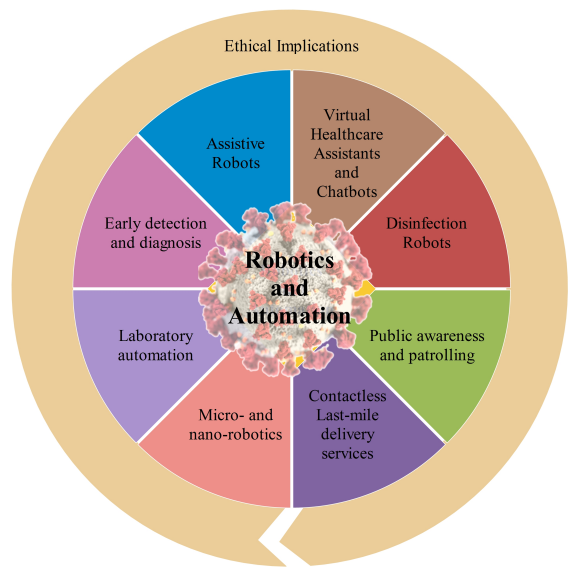

Figure 1

Robotics and Automation in Pandemic Fight.

enlisted new and rising technologies, COVID-19 made its mark on the existing medical-surgical robotic market as well, leaving companies delayed, defunct or emerged due to the new sterility, distancing and patient safety/staff requirements and standards [5].

The article is organized as follows. Section 2 describes the role of R\&A in early detection and diagnosis. Potential applications of micro-, and nano-robots in early diagnosis, monitoring and targeted drug-delivery are discussed in Section 3 Hospital and laboratory supply chain robots for handling and transportation of samples and contaminated materials are presented in Section 4 . Section 5 covers teleoperated and autonomous robots for hospital disinfection and disinfection of public spaces. The role of assistive robots in supporting humans during the quarantine and in helping them to return to work or companies to continue to function is presented in Section 6 , followed by the introduction virtual healthcare assistants and chatbots as another form of automating healthcare. Robots used by public safety and public health departments for quarantine enforcement and public service announcements are described in Section 8 followed by presenting drug, medical supplier, food/grocery delivery robots and last-mile delivery services in Section 9 The ethical implication of R\&A related to COVID-19 are discussed in Section 10. Finally, conclusions are summarized in Section 10.

\section{Early Detection and Diagnosis}

Early detection of the disease and early isolation of the affected are essential. Detecting and tracking transmission on a large scale in a timely manner, and publishing the data of infected and suspected infections are crucial in prevention and control measures, to minimize further transmission. There are numerous effi- 
cient approaches to tackle this complex task, from monitoring through AI-driven smart wearable medical devices, to smartphone apps, all the way to telemedicine and telehealth.

\subsection{Physical Sampling}

A large number of anti-epidemhic methods focus on early detection and isolation, requiring R\&A solutions for scalability. Such robots are considered to be medical devices, therefore their regulatory and clearance procedure takes time [6]. Current solutions mainly represent two distinct technical solutions, expressed in their Level of Autonomy (LoA), a scale 0-5 getting applied to the robotics domain more recently [7]:

Brain Navi's NaoTrac neurosurgical navigation robot, which has already been used in over a dozen clinical cases was retrofitted to be able to take nasal swabs for testing autonomously (LoA 4). Thus process normally requires a healthcare professional in full personal protective equipment (PPE). Lifeline Robotics, a spin-off of University of Southern Denmark has developed a similar technology, already available for pre-booking. Similar functionalities are offered by systems developed at the Shenyang Institute of Automation, China and the South Korean Institute of Machinery and Materials, Korea (in a teleoperated robotic setup-at LoA 2) (Fig. 2).

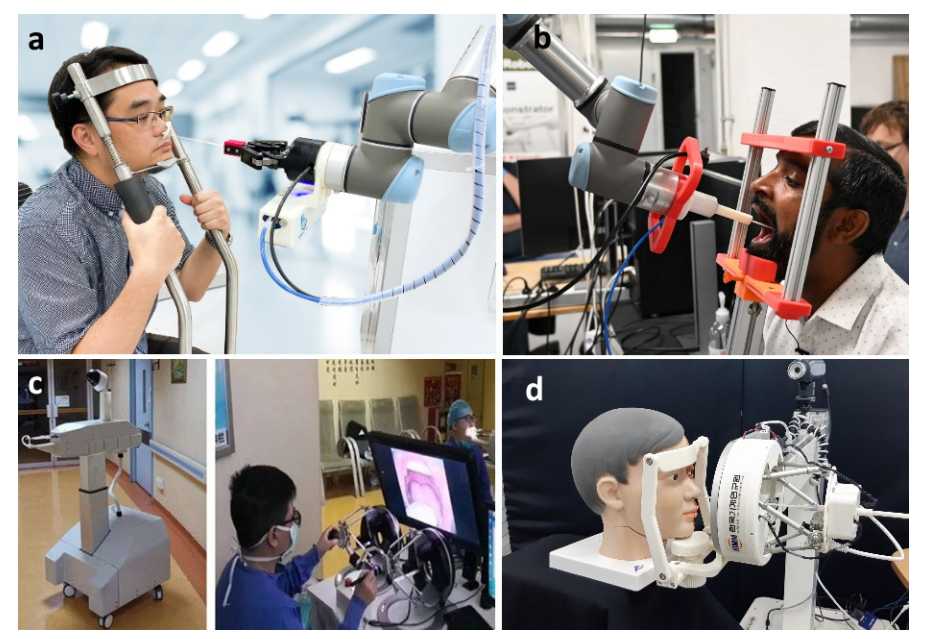

Figure 2

Robotic solutions for automated on teleoperated swabbing: a) Brain Navi; b) Lifeline Robotics; c) Shenyang Institute of Automation; d) South Korean Institute of Machinery and Materials.

Jerusalem's Hadassah Medical Center reportedly use laboratory robots to aid the management of test samples [8]. The number of samples tested has risen by two orders of magnitude, thanks to the custom designed robots by Roche, Tecan and Qiagen. Robots work jointly to extract the RNA of the virus, processing than 
$95 \%$ of the required tests.

A self-administered robot that automates the taking of COVID-19 swab tests has been developed in Singapore, helping to reduce healthcare workers' risk of exposure to the coronavirus. The swabbing process is completed in about 20 seconds, while it remains self-administered: patients can activate and terminate the process at will [9].

\subsection{Remote Monitoring}

Scaling up in non-invasive monitoring is critical as well. In Japan, SoftBank's Pepper robot has been employed for health checks and temperature monitoring at offices and public places. In China, pandemic drones petrol equipped with temperature and computer vision tool, which are used to detect symptoms of an infectious respiratory disease. Recently, drones have been used to monitor temperature sensors, heart and respiratory levels from a distance up to $10 \mathrm{~m}$, and can detect coughing and sneezing. This may provide researchers with a clear view of infections in public areas and other crowded places such as airports and health care facilities. A drone equipped with infrared thermal imaging lens takes the temperature measurement of each resident in a building without contact, and sends the data back to the disease control center. Once an abnormal body temperature is detected, medical staff will pinpoint the location of the suspected patient [10]. In addition to human body temperature monitoring, health codes have been widely used since late February in China. Each citizen receives a unique online electronic QR code, used for building a database and monitoring health history and status (Figure 3). These measures have serious ethical implications, as discussed in Section 10

Monitoring data platforms collect information uploaded by medical robots in various communities and store data (time and location of examination for suspected cases). Data mining (mines potential cases based on recorded data such as temperature, reagents, pulse, blood routine, location, etc.), data analysis and calculation (statistical analysis of the epidemic of the entire region) is also commonly performed [12]. Forecasts of the region in the next few days are given, and timely feedback is given to the epidemic units and personnel to facilitate corresponding control decisions. Some diagnostic platforms obtain real-time information through monitoring data platforms.

\section{Micro- and Nanorobotics}

Considering the current disastrous situation of COVID-19, micro- and nanorobots currently under development will be most useful for drug delivery to several targets inside human body by loading the drug dose to the pharmacyte. Then, it can transport safely to the target and release the dose by nano-injection or progressive cytopenetration. Second major use of nanorobots is body surveillance or 


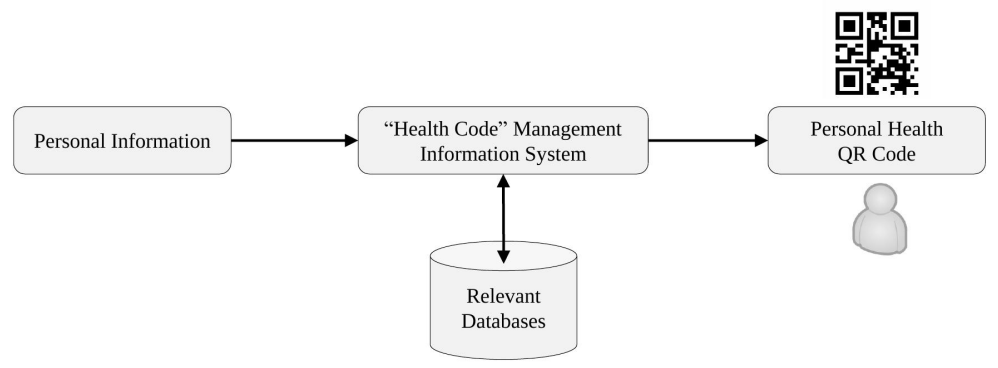

Figure 3

Personal Health QR-based System. The "health code" management information system is based on true data, and connects to relevant databases such as key personnel's dynamic control lists, and issues "health codes" based on the verification and comparison results of the mass declaration information and background data [11].

continuous monitoring of vitals, this provide a frequent response for a detected abnormal behavior. During local drug delivery, the Atomic Force Microscope (AFM) nanorobot sample tip can be used by an antibody or ligands attached to it. Such application can enhance in vitro local drug delivery research into the effects of these chemicals. Two key methods for supplying an AMF tip with certain chemical substances are available. The direct method is dipped into the specified chemical solution by the cantilever or the tip. The commonly recognized approach is the application of a connector molecule that improves the exact targeting in 3D between the AMF tip and the targeted molecule. The idea of this approach is that the chemical and the linker molecule are covalently related [13]. Major challenge of the field is to ensure safety, given the size of the robots, only LoA 4-5 systems could be deployed.

Monitoring the cellular behavior of infected persons by COVID-19 can be also done employing AFM technology. Since the AFM has a tip of about $20 \mathrm{~nm}$ or less that can be used for live cell samples; a nanorobot based on AFM may use this tip for accurate operations. Given the many advantages provided by AFM technology, such as high-resolution imagery and vacuum free operating environment, creation of nanobots based on AFM would allow imaging on a nanoscale, precise measurements of mechanical properties, nanoscale tests and the precision of movement control on living cells [13]. 


\section{Laboratory and factory automation for contagious materials}

The clinical management of coronavirus related materials and data includes the processes of anticipation, early detection, containment and mitigationeradication. Predictive AI models are urgently needed for different epidemic stages [14].

- In the early stages of a new disease, the R\&A community needs to propose methodologies for rapid deployment of technology, to make data collection, model training, testing and wide deployment efficient.

- During the containment and mitigation of COVID-19, data have become increasingly available. It is critical to develop training data sets and accurate AI models that can respond to urgent clinical needs. The AI-assisted diagnostic tools must achieve high diagnostic accuracy in order to provide added value above and beyond existing clinical tools for containment and mitigation.

- During the latter, eradication stage, there is still a need for real-time, convenient and sensitive screening tools and models to control transmission and recognize potential outbreak centers. AI models will play a key role in the critical clinical research during the post-acute phase of COVID-19.

IBM Research Europe researchers built a platform-Deep Search-that could help speeding up the process [15]. The cloud-based platform processes COVID19 literature and labels the data, table, image, and paragraph, before translating scientific content into a uniform, searchable structure.

\subsection{Wearables}

AI helmets and AI-powered glasses are used by the Chinese police to identify faces of vehicle occupants and license plates. Alerts are triggered if the vehicle occupant's information can be found in the database of confirmed cases [16]. A smart wearable medical device can be used to obtain a person's body temperature, breathing and heartbeat status, whether or not wearing a mask, and update the position information and infection/suspected infection information in real time. At the same time, the precise position of the device and the path of the terminal can be used to calculate a safe and low-risk travel path for people traveling in real time. Smart wearable medical devices can be useful in the early stage of major infectious disease outbreaks, such as screening population, quickly performing nucleic acid detection, leading to early detection and isolation. The composition of such a wearable medical device is shown in Fig. 4, which is an early concept and prototype developed under the National Nature Science Foundation of China (NSFC) program No. 7204102194.

The three major functions of this system are listed below.

1. Outbreak Visualization: based on individual body temperature charts, spatiotemporal dynamics of personnel mobility, people gathering heatmap, 


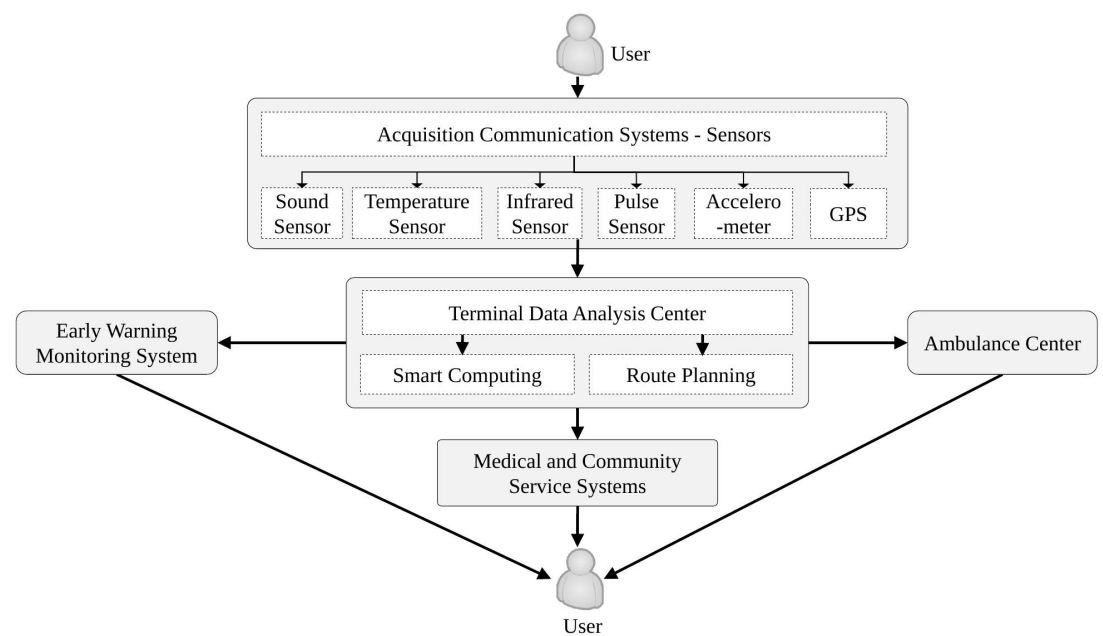

Figure 4

The composition of smart medical wearable devices, which can real-time grasp each person's current body temperature, heartbeat, breathing, confirmed infectious disease status, whether they are in contact with a confirmed/suspected infectious case, whether a mask is worn during going out, the epidemic situation during the period and so on. This information is uploaded to the terminal in real time to generate a spatiotemporal map of the epidemic situation, which quickly, multi-dimensionally and comprehensively displays the current body temperature, breathing, and heartbeat information of the personnel.

spatiotemporal map of confirmed cases;

2. Early Detection and Early Warning: screen out suspicious cases in due time;

3. Pinpoint and Route Planning: propose safe travel paths for travelers.

There are some data-driven approaches to diagnose patients through CT chest scans. Li and Qin, et al [17] proposed a 3D deep learning framework to detect COVID-19 using chest CT, named COVID-19 detection neutral network (COVNet). University of Waterloo's VIP Lab develops a COVID-Net: a convolutional neural network for COVID-19 detection via chest radiography (13,800 chest XRay of 13,725 cases from around the world. In China, there is a supercomputer that provides doctors around the world with free access to an artificial intelligence diagnostic tool for early identification of COVID-19 patients based on a chest scan. The AI system on the Tianhe-1 computer can go through hundreds of images generated by computed tomography (CT) and give the diagnosis in about 10 seconds. Wang et al [18] proposed an innovative method that did not require specialized medical imaging equipment and used footage from Kinect depth cameras, in order to identify respiratory patterns of patients. They first apply a GRU neural network with bidirectional and attentional mechanisms (BI-AT-GRU) to classify six clinically significant respiratory patterns. 


\subsection{Telehealth}

Even beyond the pandemic, emergency response can be greatly enhanced by exploiting the strengths of telehealth, particularly in the case of biological or environmental hazards. Telehealth enables remote care and attending practice, as well as it provides rapid access to information through telecommunication. During the disease outbreak, Singapore has successfully used chatbots to accelerate information flow, while video consultations and remote patient monitoring have been available in the USA since the beginning of the pandemic [19].

There were several occasions in recent history, when government agencies used telehealth solutions to increase healthcare efficiency during large-scale emergencies. The Multinational Telemedicine System developed by the North Atlantic Treaty Alliance (NATO) was deployed multiple times during military missions and crises, partially by deploying portable telemedicine devices. Owing to the coordinated work of private telemedicine companies, remote care was provided to relocated families during the devastating hurricanes Irma and Harvey. In 2003, as a result of the Severe Acute Respiratory Syndrome (SARS) pandemic, China has initiated multiple research project on using telehealth in similar situation. As a response to COVID-19, China is actively providing various remote health services. These include counseling, supervision and monitoring, even training, held by government and academic agencies. Sociometric reports showed that during the quarantine, online support and services were popular among the population, favoring the transition to a more efficient, technologically evolved form of patient care [20].

\section{Disinfection Robots}

Disinfecting surfaces and the environment, performed routinely in hospitals, has always been essential for infection prevention and control. Numerous AI-based hand hygiene assistance and monitoring solutions have appeared for personal and professional use, such as the Semmelweis Hand Hygiene System (HandInScan Zrt.) and SureWash (Goleta Ltd.) [21]. Beyond regular surface wiping with highly efficient detergents, a key strategy has been to allow the active ingredient to spread to all areas of the room, however this also needs complete lockdown and heavy air ventilation. UV-C irradiation $(200-280 \mathrm{~nm}$ ) is also an efficient disinfectant, if the radiation reaches all critical surfaces. Robots are considered as an effective technology for disinfection, preventing any harm to humans. There are two major types of applications: indoor and outdoor.

\subsection{Outdoor Disinfection Robots}

Disinfection usually means the spraying of an active material $(\mathrm{NaClO}$, sodium hypochlorite in a very low concentrate). While scientists argue that this method has very limited effect on the spread of a virus [22], engineers have developed 
both teleoperated and autonomous ground and aerial vehicles (Fig. 5). Several drone manufacturers have modified their agricultural models in order to spray disinfectant over large areas. These disinfecting drones have been used for the first time in Europe. They have been deployed in China, Chile, Indonesia, the Philippines, Colombia and the United Arab Emirates. XAG Robot has deployed disinfectant-spraying robots and drones in Guangzhou, China. Nevertheless, safety of such systems remains a major concern, and some companies have recommended similar fallback options for these systems that are being developed for self-driving cars [23] since the drones can either fly remotely controlled (LoA 2) or fully autonomously (LoA 4-5).
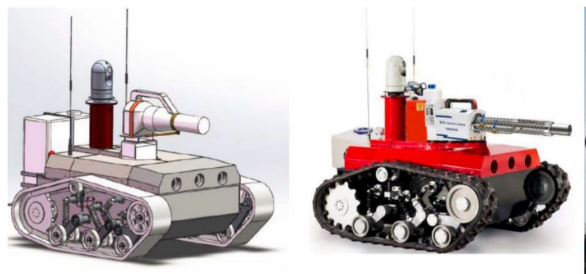

Figure 5

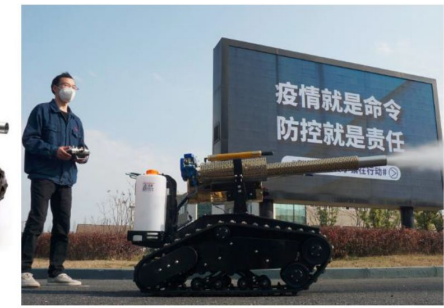

$+2$ . 


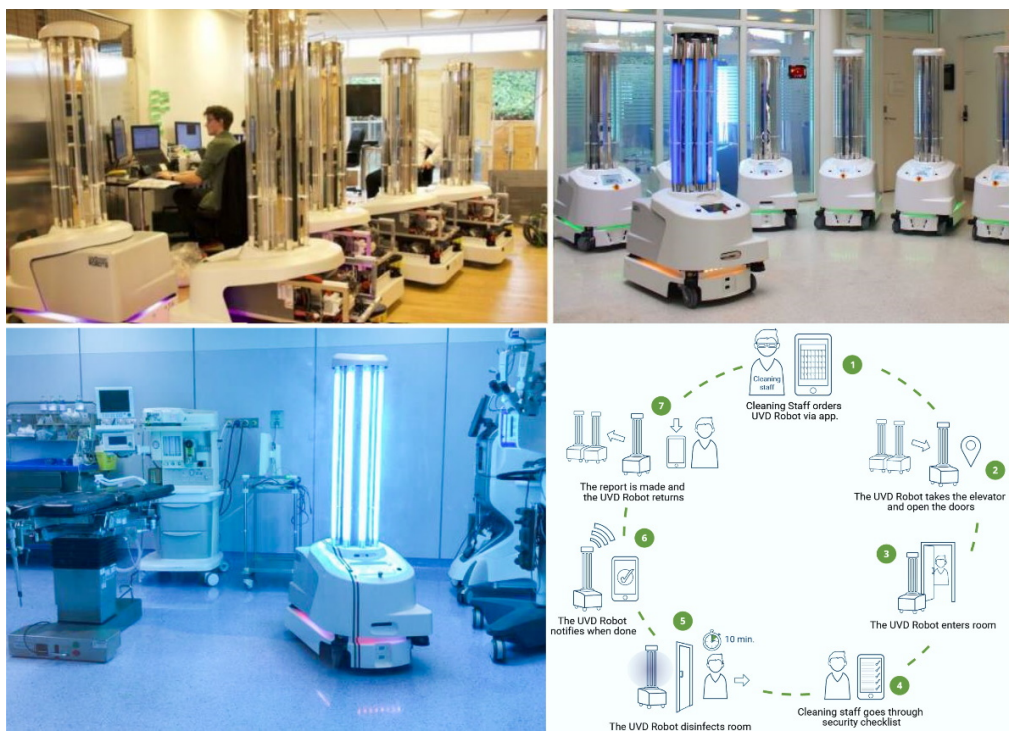

Figure 6

Indoor disinfecting robot UVD, consisting of a mobile base equipped with multiple LIDAR sensors and an array of UV lamps mounted on top. Credit: UVD Robots.

opment from Rovenso targets directly offices and commercial spaces with its focused UV-C disinfectant robot. These are typically LoA 3-5 applications. The Grainger College of Engineering at University of Illinois Urbana-Champaign's Health Care Engineering Systems Center built a low-cost, easily available robot (UVBot) that cleans spaces using UV light. UVBot has proven to be efficient in virus inactivation, the disinfection exposure time and distance for inactivation was tested using an RNA virus similar to COVID-19 [24].

\section{Assistive Robots}

Robots can perform many assistive tasks during the pandemic to mitigate the risk to healthcare professionals. Applications of assistive robots include, but are not limited to: medical care, nursing, patient monitoring, performing lab work, cooking and serving medication, or meal delivery to patients in isolation wards. Several assistive telecare robots, community medical robots and telemedicine services are used during the outbreak, e.g., doctors use the mounted iPad on Spot to remotely interact with coronavirus patients, ask them question in order to assess the patients. Fig. 7 shows the three major classes of community medical robots. Some more simplistic solutions (e.g., RoboAds) include only a mobile robot platform for autonomous positioning of kiosks on the one hand to reach the target audience with interactive (touchless) billboards, while taking telemetry 
and collecting data on people's health condition (primarily body temperature).

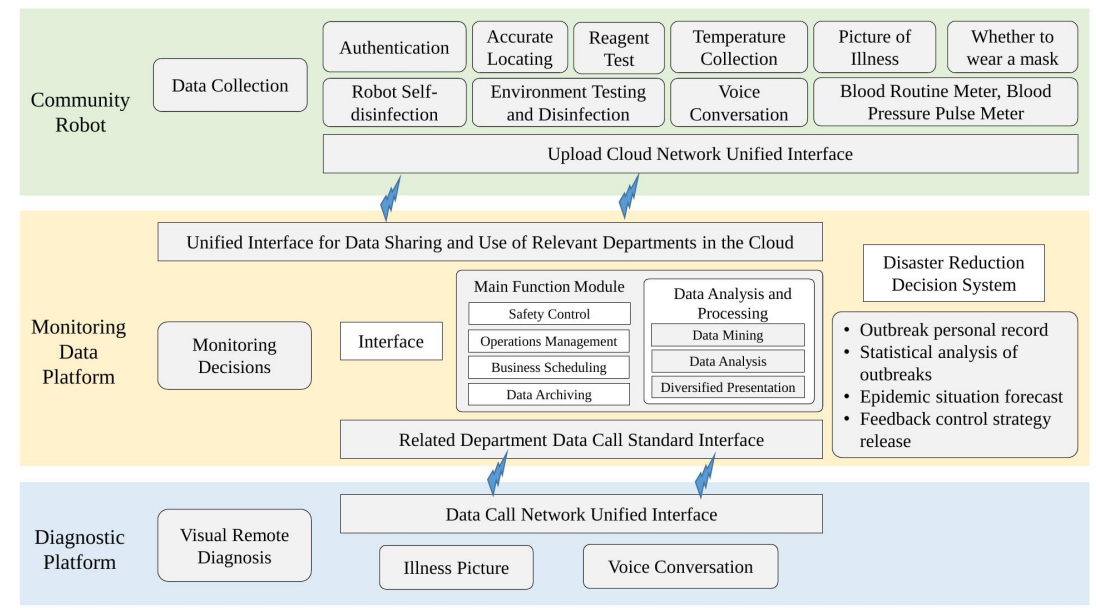

Figure 7

Community Medical Robot types employed in the fight against COVID-19.

Tommy helped Italian medical teams in treating COVID-19 patients (Fig. 8). Fig. 8 shows a remote medicine delivery for the patient using the teleoperated robot in the first affiliated hospital of Zhejiang University School of Medicine (FAHZU). This self-designed teleoperation and telepresence robot system of Zhejiang University is approved and undergoing clinical trials in the Intensive Care Unit (ICU) of FAHZU [25, 26].

The remote daily checkups are conducted via the tablet PC in the front of the robot, which allows the healthcare workers to communicate with the patients with no physical contact (Fig.9 Left). The teleoperated robot system can also be applied in non-contact interactive diagnosing and medical instrument operating (Fig. 9 Middle and 9 Right), and its functions are undergoing further improvement and validation.

Boston Dynamics has also demonstrated a quadruped robot, Spot (Fig. 10), designed by MIT and Brigham and Women's Hospital, MA. The robot is set to be used as a telepresence-based extension for healthcare workers at remote hospital areas, such as medical tents [27]. Authors argue that compared to a wheeled assistant systems, concrete bumps and curbs are not posing a challenge for a legged robot.

For the past eight months, Boston Dynamics has been trying to find ways in which their friendly yellow quadruped, Spot, can provide some kind of useful response to COVID-19. The company has been working with researchers from MIT and Brigham and Women's Hospital in Massachusetts to use Spot as a telepresence-based extension for healthcare workers in suitable contexts, with the goal of minimizing exposure and preserving supplies of PPE. 

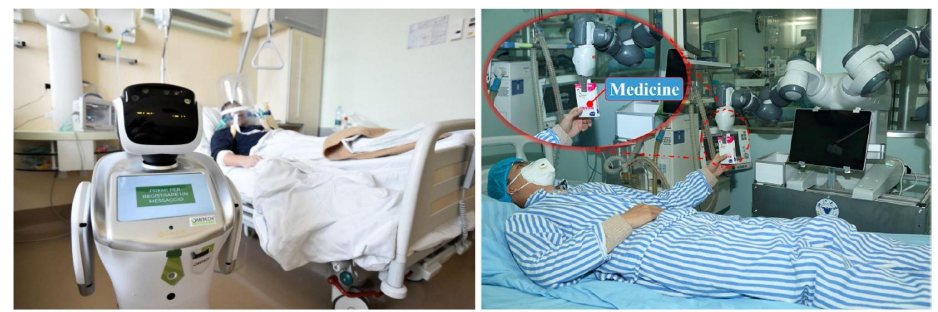

Figure 8

Left: Tommy, the robot nurse. Credit: Flavio Lo Scalzo/Reuters. Right: Remote Medicine Delivery Robot of Zhejiang University School of Medicine (FAHZU). Credit: Zhejiang University

Saskatchewan Remote Presence Robots (Fig.11) are used to deliver health care to rural and remote regions of Saskatchewan, Canada in order to protect physicians on the front lines fighting the COVID-19 pandemic.

The system illustrated in Fig. 12 uses artificial intelligence, big data, robots, the Internet of Things, modern medical technology, cloud computing and other platforms and methods.

According to the users and scenarios, it can be divided into three parts, namely, community robots, the monitoring data platform and the diagnostic platform [12]. Additionally, the RP-Vita platform from iRobot is equipped with the capability of autonomous navigation, which allows a remote clinician or bedside nurse to send the RP-VITA to a target destination with a single click.

Each community entrance can be equipped with several robots, and the position of each robot can be accurately obtained. For each person entering and leaving the community, the identification is verified and physiological data is collected. Blood test can be performed to determine whether a face mask is necessary. For suspected cases, one can further use the pneumonia test kit on the community robot and take pictures of the corresponding disease location. The medical staff is also connected to the remote diagnosis platform through the voice dialogue system. The robot will upload information about the suspected patient to the monitoring data platform, and will record and send data to the monitoring personnel. The robot can self-sterilize at regular intervals and sterilize the air in the surrounding environment and effectively prevent and reduce cross-infection.

At the same time, it can make video and voice calls with the suspects detected by the community robot. It can also check and analyze the photos of the suspects in 

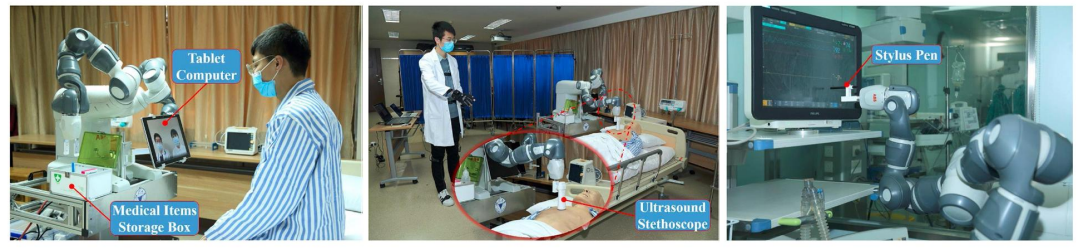

Figure 9

Left: Interactive daily consultation of physical and mental conditions using the telepresence system. Credit: Zhejiang University. Middle: Non-contact auscultation using a Doppler Ultrasound Stethoscope based on the teleoperated robot. Credit: Zhejiang University. Right: Remote operation of the medical instruments using the teleoperated robot. Credit: Zhejiang University.

order to provide medical care.

\section{Virtual Healthcare Assistants and Chatbots}

Virtual healthcare assistants and chatbots are another forms of automating the way of providing healthcare. WHO recognizes innovative solutions to reduce the need for personal contact between medical professionals and patients, and to increase accessibility to healthcare services [28]. Virtual healthcare assistants and chatbots (VHAC) are AI and rule-based systems that interact with humans to perform various tasks, to name a few, banking, answering phone calls, performing data entry, etc. These assistants use cognitive technologies such as machine learning, natural language processing (NLP), and ML to enable interactive communications with the end-users. Virtual assistant technology in the healthcare industry can assist in transforming various health processes and improve healthcare delivery worldwide. VHAC can be considered as the optimum tool to provide healthcare services to vulnerable groups such as the elderly with low immunity and co-morbidity. They can perform regular checkups and answer questions regarding health issues [29]. Recent reports claim that the global healthcare virtual assistant market is expected to grow at a rate of $24.7 \%$ from 2018 to 2025 to reach $\$ 1.7$ billion by 2025 [30].

Virtual assistants have been employed as a communicative agent. Researchers report that the usage of telemedicine increased regularly between 2005 to 2017 . 


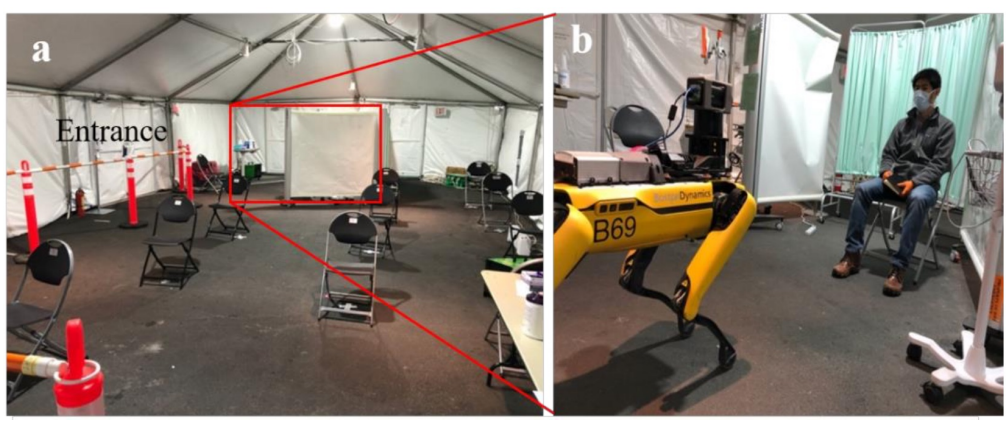

Figure 10

A medical tent outside of the emergency department of the COVID-19 triage area of the Brigham and Women's Hospital (a), and the Spot system using an IR camera for fever screening and respiratory rate detection (b). Credit: Boston Dynamics.

There is a strong demand of more robust and efficient methods and standards for evaluating these technologies [31, 32].

The need for implementing assistive technologies to fight COVID-19 has increased. Centers for Medicare \& Medicaid Services (CMS) has permitted people to use interactive applications to visit virtually their physician for a variety of purposes [33]. Canada, the UK, Italy, South Africa and India are some other countries that have deployed virtual health services. Researchers, however, suggest further enhancing the capabilities of technology. "Virtual clinics" enables virtual visits with imaging data (e.g., chest X ray) to be remotely examined [34]. Telemedicine can help establishing equality among all people as well as reducing the number of patients in clinics and healthcare centers [33, 34].

In addition, virtual healthcare assistants can play a major role in preventive measures. "Chatbot" can help screen or identify early symptoms, teach and train users essential hygienic practices (e.g. "Amplify.ai", and "Facebook's Messenger for COVID-19 Program") [35]. Another chatbot designed specifically for COVID-19 is "the Coronavirus Self-Checker" [36], which provides resources to protect people and advises users whether or not they need to visit medical care centers.

\section{Public Awareness and Patrolling}

Linking to the previous Section, it is obvious that voice assistants can be widely used, being able to complete 200 calls in 5 minutes and offer a statistical result. With the trial of voice AI-agents, the efficiency of epidemic investigation has been greatly improved.

UAVs have been widely used for the fight against COVID-19. Drones can be equipped with high-power speakers and warnings can be broadcasted to the crowd 


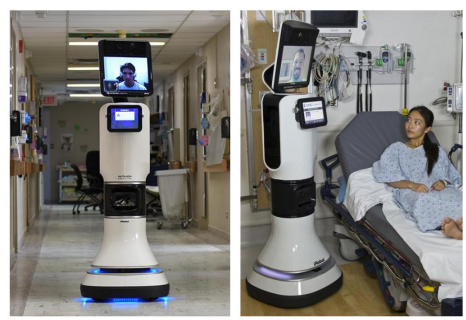

Figure 11

Left: Saskatchewan Remote Presence Robots. Credit: University of Saskatchewan and Saskatchewan Health Authority Remote Presence Robotic Program. Right: Remote Presence Virtual + Independent Telemedicine Assistant (RP-VITA). Credit: iRobot.

or to people not wearing masks. At the same time, drones can detect sick people quickly through thermal imaging and target recognition systems.

Another example is Boston Dynamics' Spot that is used at parks to remind visitors of social distancing measures or patrol at lockdown/curfew areas.

Another example is Knightscope Robotics that can be used for patrolling corporate facilities. Robots are used for remote monitoring of streets and corporate campuses (Figure 13).

\section{Contactless Last-mile Delivery Services}

Reducing physical contact during the pandemic demands last-mile delivery (LMD) robots. Last-mile is a term used in supply chain management and transportation planning to describe the movement of people and goods from a transportation hub to the destination [37]. Various innovative solutions for LMD had been or tested to reduce the delivery cost, increase customer satisfaction and minimize the negative environmental impact. These solutions include, but are not limited to, cargo-bikes, semi and fully autonomous LMD, delivery Droids (Bots), EPlaette, Postal delivery, driverless deliveries and privately-owned AV. According to Makinsey, semi and fully autonomous LMD will reduce costs by approximately $10-40 \%$ [38].

Contactless LMD systems and services can result in avoiding physical contact between caregivers and patients or between delivery workers and the recipients. These benefit from the rapid proliferation of connected technologies and the recent advancements in semi and fully autonomous delivery platforms which revolutionize the urban logistics and provide a safe and efficient delivery methods 


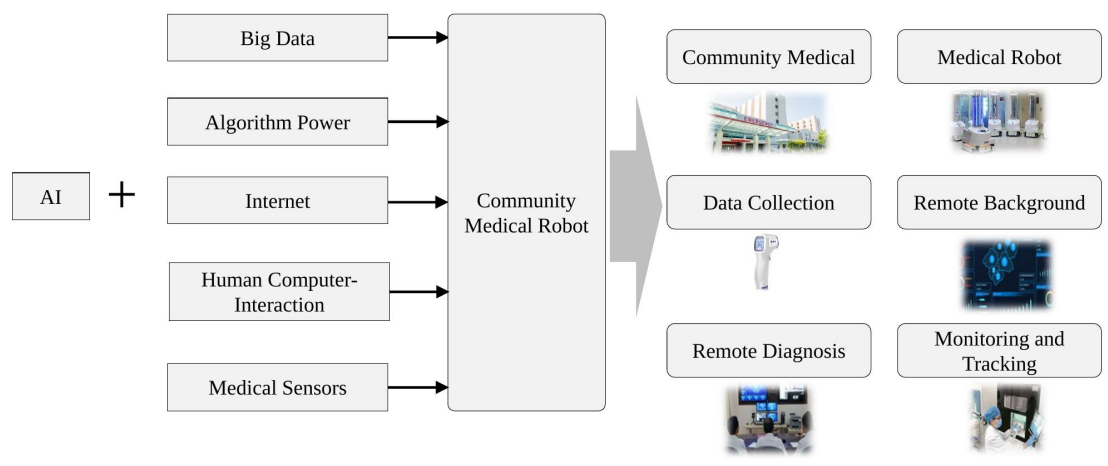

Figure 12

A composition framework diagram of community medical robots.

for medical supplies [39], medications [40], food, grocery and other goods [41. 42]. Fig. 14a shows AIMBOT38, an autonomous mobile robot developed by UBTECH Robotics, performs disinfection tasks at Shenzhen Third Hospital.

The demand for food delivery facilities has never been higher as most restaurants and cafés are closed during lockdown. Fig. ??b shows Nuro driverless vehicle that has been approved for delivery tests in California in April 2020 [42]. Drone distribution of medication and Personal Protective Environment (PPE) is a valuable option as drones can easily carry samples from hospitals to labs, provide simple security services, and bring medical equipment to patients' homes such that immediate control can be provided. Currently some companies in China and Ghana are actively using this technology.

Shortage of PPE and other medical equipment was a global issue in the first half of 2020, until production volumes could be increased, but some critical issues remain. WHO estimates that one in every six COVID-19 patients needs breathing support through medical ventilators at intensive care units (ICUs). Robots could help in supplies in several ways [43]:

- Increasing medical ventilator production by automating approximately $40 \%$ to $50 \%$ of assembly tasks;

- Re-purposing existing non-ventilator production-such as airplane, jet engine, automobile, and vacuum production - to ventilator production;

- Reducing the need of human operators;

- Maintaining social distancing at manufacturing facilities;

- Maintaining ability for future production. 


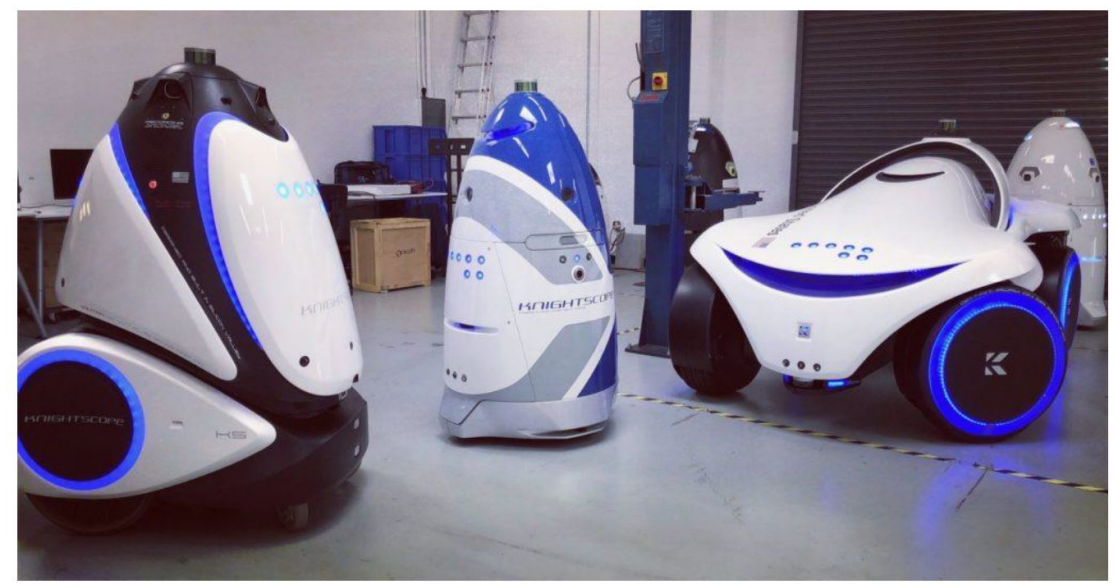

Figure 13

The Knightscope lineup of indoor and outdoor telepresence robots. Credit: Knightscope Robotics.
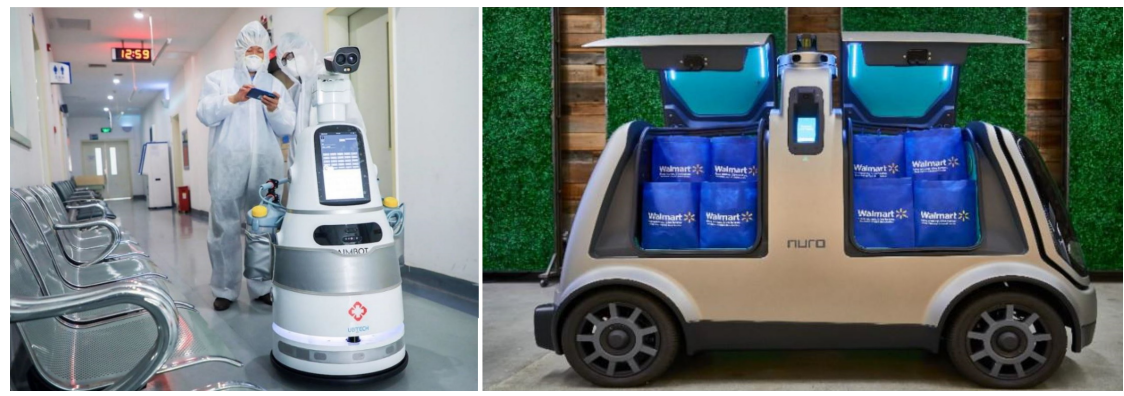

Figure 14

a) AIMBOT, an autonomous mobile robot. Credit: UBTECH Robotics. b) Nuro driverless vehicles. Credit: Nuro.

These functions may apply to other products in need as well.

\section{Ethical Implication of Robotics and Automation}

Previous Sections highlighted how important Robotics and AI are for addressing COVID-19. At same time, they can pose serious risks for our fundamental rights ranging from the impact on democracy to our right to life [44], [45].

Currently, several Governments are establishing partnerships with private companies to get access and track people's behavior and location. In [46], one can see a live index elaborated by Top10PVN, about the use of cell phone location per country, which ranges from processing anonymized data to the tracking of an 
individual person and their contacts. Governments argue that mobile location can provide invaluable information to help understanding the dynamic of COVID-19 and, thus, reducing public threats. The data collected can be used to identify patterns of movements and collect inform on how the virus is being spread in a particular region or, even, at individual level, alert authorities if a particular individual is obeying to home quarantine [47].

The main concern is that these can open the door to further, wider use of such technology, increasing the surveillance of the citizens under different and questionable justifications. This raises serious concerns about potential abuse that would directly impact our privacy, freedom of expression and freedom of association accentuating, e.g., discrimination and marginalization of groups. Recently, a joint statement signed by different organizations urged Governments ensure the use of digital technologies to address the pandemic is in line with human rights [48]. Governments should observe that

- all surveillance measures must be lawful, necessary and proportionate;

- all monitoring and surveillance powers must be time-bound;

- all monitoring may only continue for as long as necessary to address the current pandemic.

Considering the latter, Amnesty International [49], emphasizes "there is a real danger surveillance measures become permanent fixtures. In the meanwhile, the mounting financial difficulties decrease the companies capabilities to deal with ethical issues and CSR related activities [50].

Another relevant issue is whether an AI-based system should ultimately make critical medical decisions (keeping in mind that trusting ADs with this also puts a huge load on them). There are numerous questions arising on what the data should be used to provide an answers, and whether age, gender, known diseases, race, social status or other should be considered.

In China, researchers developed an AI-based tool to assist doctors in making choices about patients with COVID-19 using blood samples [51]. However, they argue that human agency should never be undermined, i.e., humans should not be obligated to use system decisions blindly without having them scrutinized. The reason is simple: AI-based systems are only math tools. They are neither intelligent nor neutral. They are fairly as good as the quality of the data that is used in their training set. This fact is corroborated with evidences presented in different reports that show gross errors produced by some systems, which a human would never make [52].

A correlated problem is that most people who will use AI-based systems do not know their limitations. This has led to situations where people in key positions anthropomorphized and over-trusted the technology, and, using fallacious arguments, imposed the adoption of these systems in critical and sensitive tasks. Therefore, initiatives like IEEE Global Initiative on Ethics of Autonomous and Intelligent Systems [53] and IEEE/MIT Global Council on Extended Intelligence [54] are very relevant in the resolution of related COVID-19 challenges. 
The related IEEE P7000 Ethically Aligned Design standard may have great importance in pandemic-related critical technology development and assessment. P7000 puts emphasis on 5 key domains: accountability;auditability; dependability; predictability and transparency.

While the P7000 aims to educate and empower people to take ethics into consideration during the life cycle of AI-based systems, the latter discusses AI in creating a new narrative for the domain to demystify the hype that surrounds it. The risk for misusing technology is high. Organizations like ProPublica recently pointed out people with disabilities may be denied from lifesaving care during the Pandemic [55]. In Brazil, the Ministry of Health suggested in a press collective [56] that saving an elderly is more financially expensive than saving a young person. Predictions like that can be incorporated in an AI-based system causing irreparable losses, and due to the opaqueness of the system, this discriminatory information would never be uncovered and the responsible would never be held accountable. These domains shall further be regulated and managed, for which we could even take inspiration from SCI-FI and literature [57].

\section{Conclusions}

The global battle against the novel coronavirus and COVID-19 has seen robotics and automation playing an extremely important roles in understanding the scope of the pandemic, dealing with the contagious nature of the virus and decelerating its spread. This article structurally reviewed a number of robotics and automation applications in the battle against COVID-19. These applications include early detection and diagnosis, assistive robots, indoor and outdoor disinfection robots, public awareness and patrolling, last-mile delivery services, micro- and nano-robotics and laboratory automation. Ethical implications of robotics and automation were also discussed in the context of the novel principles of ethically aligned design.

Many conclusions can be drawn from this unprecedented crisis that has profoundly impacted humanity on a global scale. There is also a need to fully understand the limitations of automated systems for not over-trusting the technology and creating false arguments especially in critical health-related tasks. Medical regulatory branches have an outstanding responsibility in this. Measures should be taken to protect digital rights, stop the surveillance once the pandemic ends, ban new totalitarianism of surveillance technology and digital totalitarian states. Robotics and AI in general have to be exploited, yet regulated and smartly directed toward the benefit of humanity.

\section{Acknowledgment}

T. Haidegger's work is partially supported by the ELKH SZTAKI - OE CyberMedical System Development project. T. Haidegger is supported through the 
New National Excellence Program of the Ministry of Human Capacities.

T. Haidegger is a Bolyai Fellow of the Hungarian Academy of Sciences, his research is partially supported by the EFOP-3.6.2-16-2017-00016 project.

\section{References}

[1] G. Hager, V. Kumar, R. Murphy, D. Rus, and R. Taylor. The role of robotics in infectious disease crises. arXiv preprint arXiv:2010.09909, 2020.

[2] World Health Organization. Report of the WHO-China joint mission on coronavirus disease 2019 (COVID-19). Technical report, 2020.

[3] C. Shorten, T. M. Khoshgoftaar, and B. Furht. Deep learning applications for covid19. Journal of Big Data, 8(1):1-54, 2021.

[4] A. A. Hussain, O. Bouachir, F. Al-Turjman, and M. Aloqaily. Ai techniques for covid-19. IEEE Access, 8:128776-128795, 2020.

[5] A. Zemmar, A. M. Lozano, and B. J. Nelson. The rise of robots in surgical environments during covid-19. Nature Machine Intelligence, 2(10):566-572, 2020.

[6] P. Barattini, F. Vicentini, G. S. Virk, and T. Haidegger. Human-robot interaction: safety, standardization, and benchmarking. CRC Press, 2019.

[7] T. Haidegger. Autonomy for surgical robots: Concepts and paradigms. IEEE Transactions on Medical Robotics and Bionics, 1(2):65-76, 2019.

[8] L. Luxner. Deluged by pandemic needs, israeli doctors get help from unlikely source: robots. Jewish Telegraphio Agency, Jul 2020.

[9] Singapore-made COVID-19 swab test robot could reduce healthcare workers' risk of infection, Sep 2020.

[10] W. Fan. Epidemic promotes surge in demand for infrared temperature measuring instruments: we "reunite families" and they "overtime". Science and Technology Board Daily., 2020.

[11] Shenzhen Municipal Affairs Service Data Administration. Epidemic pass reference architecture and technical guide, 2020.

[12] S. Han and Y. Fan. Discussion on the application of logistics robot distribution in community epidemic isolation. Digital Communication World, 2020.

[13] B. Song, R. Yang, N. Xi, K. Patterson, C. Qu, and K. Lai. Cellular-level surgery using nano robots. J Lab Autom., 17(6):425-434, 2012.

[14] Y. Hu, J. Jacob, G. Parker, and et al. The challenges of deploying artificial intelligence models in a rapidly evolving pandemic. Nature Machine Intelligence, (2):298-300, 2020.

[15] L. Peskoe-Yang. Ibm's new ai tool parses a tidal wave of coronavirus research. IEEE Spectrum, 1 Jul 2020.

[16] Q. Sun. AR glasses check the car, induction disinfection. Technical report, 2020.

[17] L. Li, L. Qin, Z. Xu, Y. Yin, X. Wang, B. Kong, J. Bai, Y. Lu, Z. Fang, Q. Song, et al. Artificial intelligence distinguishes covid-19 from community acquired pneumonia on chest CT. Radiology, 2020.

[18] Y. Wang, M. Hu, Q. Li, X.-P. Zhang, G. Zhai, and N. Yao. Abnormal respiratory patterns classifier may contribute to large-scale screening of people infected with covid-19 in an accurate and unobtrusive manner. arXiv preprint arXiv:2002.05534, 2020.

[19] A. C. Smith, E. Thomas, C. L. Snoswell, H. Haydon, A. Mehrotra, J. Clemensen, and L. J. Caffery. Telehealth for global emergencies: Implications for coronavirus 
disease 2019 (COVID-19). Journal of telemedicine and telecare, pages 309-313, 2020.

[20] X. Zhou, C. L. Snoswell, L. E. Harding, M. Bambling, S. Edirippulige, X. Bai, and A. C. Smith. The role of telehealth in reducing the mental health burden from COVID-19. Telemedicine and e-Health, 26(4):377-379, 2020.

[21] T. Haidegger, V. Varga, A. Lehotsky, P. Róna, R. Pethes, P. Szerémy, L. Szilagyi, T. Ferenci, and L. Kovács. Information technology tools employed in infection control. In 16th IEEE International Symposium on Computational Intelligence and Informatics (CINTI), pages 339-344, 2015.

[22] R. F. Service. Does disinfecting surfaces really prevent the spread of coronavirus? Science Magazine, Mar 2020.

[23] D. A. Drexler, A. Takács, T. D. Nagy, and T. Haidegger. Handover process of autonomous vehicles-technology and application challenges. Acta Polytechnica Hungarica, 16(9):235-255, 2019.

[24] University of illinois urbana-champaign develops autonomous robot to kill covid-19. Healthcare Hygiene Magazine, OCT 2020.

[25] G. Yang, Z. Pang, M. J. Deen, M. Dong, Y. Zhang, N. H. Lovell, and A. M. Rahmani. Homecare robotic systems for healthcare 4.0: Visions and enabling technologies. IEEE Journal of Biomedical and Health Informatics, 2020.

[26] Z. Pang, G. Yang, R. Khedri, and Y.-T. Zhang. Introduction to the special section: convergence of automation technology, biomedical engineering, and health informatics toward the healthcare 4.0. IEEE Reviews in Biomedical Engineering, 11:249-259, 2018.

[27] H.-W. Huang, C. Ehmke, G. Merewether, F. Dadabhoy, A. Feng, A. J. Thomas, C. Li, M. d. Silva, M. H. Raibert, E. W. Boyer, et al. Agile mobile robotic platform for contactless vital signs monitoring. 2020.

[28] World Health Organization. Telemedicine: opportunities and developments in member states. report on the second global survey on eHealth., 17(6):425-434, 2009.

[29] C. Kuziemsky, A. J. Maeder, O. John, S. B. Gogia, A. Basu, S. Meher, and M. Ito. Role of artificial intelligence within the telehealth domain: Official 2019 yearbook contribution by the members of imia telehealth working group. Yearbook of medical informatics, 28(1):35, 2019.

[30] Meticulous Research. Healthcare virtual assistant market - global opportunity analysis and industry forecast (2018-2025). page 103, 2019.

[31] S. Hoermann, K. L. McCabe, D. N. Milne, and R. A. Calvo. Application of synchronous text-based dialogue systems in mental health interventions: systematic review. Journal of medical Internet research, 19(8):e267, 2017.

[32] L. Laranjo, A. G. Dunn, H. L. Tong, A. B. Kocaballi, J. Chen, R. Bashir, D. Surian, B. Gallego, F. Magrabi, A. Y. Lau, et al. Conversational agents in healthcare: a systematic review. Journal of the American Medical Informatics Association, 25(9):1248-1258, 2018.

[33] P. Webster. Virtual health care in the era of COVID-19. The Lancet, 395(10231):1180-1181, 2020.

[34] D. S. W. Ting, L. Carin, V. Dzau, and T. Y. Wong. Digital technology and COVID19. Nature medicine, 26(4):459-461, 2020.

[35] E. El Habre. A case for switching from self-fulfilling prophecies to rights in ai journalism. 2020.

[36] L. Town and K. Hoffman. Coronavirus and the Caregiver. Omega Press, 2020.

[37] R. W. Goodman. Whatever you call it, just don't think of last-mile logistics, last. Global Logistics \& Supply Chain Strategies, 9(12), 2005. 
[38] M. Joerss, J. Schröder, F. Neuhaus, C. Klink, and F. Mann. Parcel delivery: The future of last mile. McKinsey \& Company, 2016.

[39] T. Niels, M. T. Hof, and K. Bogenberger. Design and operation of an urban electric courier cargo bike system. In 201821 st international conference on intelligent transportation systems (itsc), pages 2531-2537. IEEE, 2018.

[40] L. Nianzhen. How a chinese drone delivery startup is capitalizing on COVID-19. Nikkei Asian Review, Mar 2020.

[41] Robot to deliver meals, medication to Covid-19 patients in S'pore. Bangkok Post, Mar 2020.

[42] S. Crowe. Nuro driverless vehicles approved for delivery tests in california. The Robot Report, Apr 2020.

[43] A. A. Malik, T. Masood, and R. Kousar. Repurposing factories with robotics in the face of COVID-19. Science Robotics, 5(43), 17 Jun 2020.

[44] A. Khamis, H. Li, E. Prestes, and T. Haidegger. Ai: A key enabler of sustainable development goals, part 1 [industry activities]. IEEE Robotics \& Automation Magazine, 26(3):95-102, 2019.

[45] A. Khamis, H. Li, E. Prestes, and T. Haidegger. Ai: A key enabler for sustainable development goals: Part 2 [industry activities]. IEEE Robotics \& Automation Magazine, 26(4):122-127, 2019.

[46] J. Morley, J. Cowls, M. Taddeo, and L. Floridi. Ethical guidelines for sars-cov-2 digital tracking and tracing systems. Available at SSRN 3582550, 2020.

[47] K. Uibu. Poland is making its citizens use a 'selfie' app during the coronavirus crisis. ABC news Australia, Apr 2020.

[48] Joint civil society statement: States use of digital surveillance technologies to fight pandemic must respect human rights. Human Rights Watch, Apr 2020.

[49] COVID-19, surveillance and the threat to your rights. Amnesty International, Apr 2020.

[50] P. Karácsony. Analyzing the relationship between leadership style and corporate social responsibility in hungarian small and medium-sized enterprises. Acta Polytechnica Hungarica, 17(7):183-198, 2020.

[51] S. Chen. Should AI help make life-or-death decisions in the coronavirus fight? South China Morning Post, Mar 2020.

[52] D. Heaven. Why deep-learning ais are so easy to fool. Nature, 574(7777):163-166, 2019.

[53] P. Winch. Ethics and action. Routledge, 2020.

[54] G. Adamson, J. C. Havens, and R. Chatila. Designing a value-driven future for ethical autonomous and intelligent systems. Proceedings of the IEEE, 107(3):518$525,2019$.

[55] A. Silverman. People with intellectual disabilities may be denied lifesaving care under these plans as coronavirus spreads. ProPublica (https://www. propublica. org/article/people-with-intellectual-disabilities-may-be-denied-lifesaving-careunder-these-plans-as-coronavirus-spreads). Accessed, 19, 2020.

[56] Teich já sugeriu que salvar idoso em vez de jovem é mais caro à saúde. (In Portugese) Carta, Apr 2020.

[57] R. R. Murphy. Robots and pandemics in science fiction. Science Robotics, 5(42), 2020. 